\title{
Prevention of progression of coronary atherosclerosis by treatment of hyperlipidaemia: a seven year prospective angiographic study
}

\author{
ESKO A NIKKILĂ, PERTTI VIIKINKOSKI, MATTI VALLE, M H FRICK
}

\begin{abstract}
The progression of coronary atherosclerosis was assessed by repeat angiography in 28 patients and 20 controls with hyperlipidaemia (serum cholesterol concentration $>7.2 \mathrm{mmol} / 1$ (278 $\mathrm{mg} / 100 \mathrm{ml})$ or serum triglyceride concentration $>2.0 \mathrm{mmol} / 1(177 \mathrm{mg} / 100 \mathrm{ml})$, or both) and symptomatic coronary artery disease of two or three vessels. Twenty eight patients (26 men and two women) were treated with diet and drugs (clofibrate or nicotinic acid, or both) to lower lipid concentrations. Twenty men taking part in a simultaneous study served as non-randomised controls. They received medical treatment for coronary artery disease but no treatment to reduce lipid concentrations. The initial levels of coronary risk factors and the angiographic state were comparable in the two groups. In the 28 patients total cholesterol, total triglyceride, and low density lipoprotein cholesterol concentrations were reduced by an average $18 \%, 38 \%$, and $19 \%$ respectively by treatment for hyperlipidaemia and high density lipoprotein cholesterol concentration was increased on average by $10 \%$. The treatment maintained these concentrations during a follow up of seven years.
\end{abstract}

By all criteria coronary lesions progressed significantly less in the patients than the controls: the angiographic state remained completely unchanged in nine $(32 \%)$ of the patients compared with only one $(8 \%)$ of the surviving controls; of the arterial segments at risk, 46 $(16.5 \%)$ progressed in the patients compared with $50(38.2 \%)$ in the controls $(p<0.001)$; and the coronary obstruction increased less in patients than in controls $(p<0.05)$. Cardiac survival was $89 \%$ in seven years in the patients compared with $65 \%$ in five years in the controls $(p<0.01)$. The anginal symptoms diminished or remained stable in 16 of the 24 patients who survived until the end of the study.

The progression of coronary atheromatosis was significantly greater in those patients who during the seven years of treatment had an average total cholesterol concentration, VLDL plus LDL cholesterol concentration, or ratio of LDL to $\mathrm{HDL}$ cholesterol concentration above the respective median value than in those with the corresponding values below median. On the other hand, the patients with HDL cholesterol concentrations above the median during treatment showed less progression than those with lower HDL cholesterol concentrations. The increase in coronary obstruction was inversely related to the average HDL cholesterol concentration during

University of Helsinki, 00290 Helsinki 29, Finland

ESKO A NIKKILÄ, MD, professor of medicine, third department of medicine

PERTTI VIIKINKOSKI, MD, internist, Hyvinkää District Hospital

MATTI VALLE, MD, physician in chief, department of radiodiagnostics M H FRICK, MD, professor of medicine, first department of medicine

Correspondence to: Professor E A Nikkilä. treatment. The progression was not, however, related to LDL cholesterol concentration during treatment.

Progression of even advanced coronary atherosclerosis may be retarded by reducing raised serum lipid concentrations and increasing HDL cholesterol concentrations.

\section{Introduction}

Experimentally produced atherosclerotic lesions in animals may regress when an atherogenic diet is stopped or hyperlipidaemia is treated. ${ }^{1-3}$ In man the effects on progression of coronary heart disease of regimens that lower plasma lipid concentrations have been assessed in several primary and secondary prevention trials; these have produced both positive ${ }^{4-8}$ and negative ${ }^{910}$ results. Direct visual follow up of the course of the atheromatous lesions has been done by sequential coronary or femoral angiography in a limited number of subjects during treatment for hyperlipidaemia. Regression of femoral atherosclerosis has been observed in many patients after effective reduction of serum lipid concentrations, even over relatively short periods. ${ }^{11-14}$ These results have recently been confirmed in a randomised controlled trial in which a $60 \%$ reduction in progression of femoral atherosclerosis was achieved with treatment that reduced lipid concentrations. ${ }^{15}$ In contrast, regression or retarded progression of coronary atheromatosis has been reported in only anecdotal cases, in most of which an extensive reduction in serum cholesterol concentrations was achieved by ileal bypass or plasmapheresis. ${ }^{1617}$ In one long term study coronary lesions were seen to become stable in eight of 12 patients with familial hypercholesterolaemia treated with a diet and colestipol. ${ }^{18}$ No systematic studies of the effect of treatment of the common forms of hyperlipidaemia on progression of coronary atherosclerosis had been published before we began this study. Recently, however, the results of a parallel prospective study have been published. ${ }^{18 a}$

In 1975 we began a prospective study to assess the effect of treatment that reduced lipid concentrations on the progression of coronary atherosclerosis in patients with raised serum cholesterol or triglyceride concentrations and with premature coronary artery disease of two or three vessels. ${ }^{19}$ Patients were followed up for seven years with triannual measurements of serum lipid and lipoprotein concentrations and with repeat coronary angiography at two and seven years. For ethical reasons a randomised placebo treated control group was not included, but we compared our results with those obtained from a simultaneous prospective angiographic study in which no intervention was made. ${ }^{20}$

\section{Subjects and methods}

PATIENTS

Patients were recruited in 1975 from Helsinki University Hospital Meilahti clinics. All coronary angiograms obtained during the preceding year for patients aged below 57 were examined. Patients with coronary artery disease of two or three vessels and with serum total cholesterol concentration exceeding $7 \cdot 2 \mathrm{mmol} / 1(278 \mathrm{mg} / 100 \mathrm{ml})$ or serum triglyceride concentration exceeding $2.0 \mathrm{mmol} / 1$ (177 mg/ 
$100 \mathrm{ml}$ ), or both, were eligible. Patients considered for surgical treatment and those with clinical diabetes, poor left ventricular function, cardiac failure, or uncontrolled hypertension were excluded. All the remaining patients were asked to volunteer for the study, the purpose and design of which were explained to them. Informed consent was obtained from 41 patients, but before the start of treatment seven withdrew their consent, three did not fulfil the entry criteria with regard to serum lipid concentration at control examination, and one died. Moreover, two patients who underwent angiography again after two years declined the final examination and were excluded. Thus the final series comprised 28 patients (table I), of whom only two were women.

\section{CONTROLS}

A non-randomised control group was formed from patients with coronary disease participating in another prospective study carried out at the same time at the same hospital. ${ }^{20}$ This other trial compared the effects of coronary bypass surgery with those of medical treatment on progression and outcome of coronary artery disease in men aged below 65 . The entry criteria had included angina pectoris, a positive exercise test, and stenoses of more than $50 \%$ in at least two coronary arteries. One hundred patients had then been randomly assigned to receive either medical treatment or bypass surgery, and the surviving subjects underwent coronary angiography again after five years. ${ }^{20}$ Among the 50 patients randomly allocated to receive medical treatment 20 men had hyperlipidaemia by the criteria given above for our study and were not receiving any diet or drugs that lowered lipid concentrations. These men served as controls for the present study. Table I gives their baseline data and shows that patients and controls were largely comparable for major risk factors and also for degree of coronary lesions at entry. For some reason more patients than controls had a history of myocardial infarction or hypertension.

\section{SCHEDULE OF TREATMENT FOR PATIENTS}

Diet and drugs were used to produce maximal reduction of the raised cholesterol or triglyceride concentrations. Patients with hypercholesterolaemia were instructed to receive a diet containing roughly $30^{\circ}{ }^{\prime}$ of energy as fat with a ratio of polyunsaturated to saturated fats of about $1: 1$. Intake of cholesterol was limited to less than $300 \mathrm{mg}$ a day. Subjects with raised triglyceride concentrations were also advised to reduce their consumption of saturated fats and to increase their intake of vegetable oils and fish. Overweight subjects received instructions for weight reducing diets. All instructions were given by a dietitian, whom the patients visited at least twice during the first year of the trial. The serum lipid and lipoprotein concentrations were measured, and the patients were seen by one of us (PV) every six weeks. If the diet did not reduce the total cholesterol concentration to less than $6.0 \mathrm{mmol} / 1(232 \mathrm{mg} / 100 \mathrm{ml})$ and total triglyceride concentration to less than $1.6 \mathrm{mmol} / 1(142 \mathrm{mg} / 100 \mathrm{ml})$ clofibrate was started at a daily dose of $2.0 \mathrm{~g}$. If the correct concentrations were still not achieved 1.5-2.5 $\mathrm{g}$ nicotinic acid/day was added.

Adjustment of drugs and diets was stopped after one year, and the patients continued with the best combination for the remaining six years. During this time the serum lipid and lipoprotein concentrations, body weight, and blood pressure were measured every four months. The blood samples were taken by local laboratories and serum was sent to the hospital for analysis. The patients were contacted each time by telephone or letter but visited the physician or hospital only if symptoms indicated medical care. Drug treatment for hypertension remained constant throughout the study. Smoking habits were not changed, but physical activity was slightly increased when the symptoms of angina permitted. Strenuous activity or sports were not, however, undertaken by any of the patients.

\section{CORONARY ANGIOGRAPHY}

Coronary arteriography was done in 1975 (before entry to the study) and after two and seven years. Coronary arteries were selectively injected in multiple projections by the Judkins technique. Nitroglycerin was given sublingually before the injections. Both $35 \times 35 \mathrm{~cm}$ films and cineangiography were used. The cut films were used for the analysis. The luminal diameter was measured with a calibrated glass gauge checked with a micrometer. ${ }^{21}$ Calculations based on geometric blur and film and folio blur showed an accuracy of $0.4 \mathrm{~mm}$ for the measurements of luminal diameters. Seven coronary arteries were analysed for each patient. In addition, the proximal, middle, and distal segments of three main epicardial arteries were analysed separately. The obstruction was estimated as the proportional reduction in the luminal diameter. The progression of coronary lesions at repeat angiography was assessed by one of us (MV) without any knowledge of the clinical or laboratory data. The progression was measured in two different ways. Firstly, the total increase in proportional luminal narrowing was calculated for each patient; thus an obstruction increasing from 50 to $75 \%$ in one vessel or segment and from 25 to $50 \%$ in another gave a total increase in obstruction of $50 \%$. Progression was said to be present if the luminal narrowing at a lesion had increased by a minimum of $20 \%$ or if a subtotal obstruction had advanced to total occlusion. Secondly, the number of major arterial segments showing progression was counted and related to the number of segments at risk for progression. Segments with complete occlusion at the first examination and those distal to the occlusion were not taken to be at risk for further progression, and their number was subtracted from the total number of arterial segments counted to get the number of segments at risk.

Initial angiography was done in 30 patients and 20 controls. Thirty patients were examined again at two years, but two declined the third angiography and were excluded. Four patients died before the end of the study; for them the results of the examination at two years were used. For all the others the assessment of progression was carried out from the results of angiography at seven years only. The surviving controls underwent repeat angiography after only five years. ${ }^{20}$ To be comparable with the patients the progression data for the controls were adjusted for seven years by assuming that the progression was a linear function of time. This assumption is supported by the findings of Bruschke et al, ${ }^{22}$ which suggest a largely linear correlation between the proportional progression in occlusion of coronary segments and the intervals between coronary angiography.

\section{ANALYSES OF LIPID AND LIPOPROTEIN CONCENTRATIONS}

Serum was obtained after an overnight fast lasting 12 hours. The major lipoprotein fractions very low density lipoprotein (VLDL), low density lipoprotein (LDL), and high density lipoprotein (HDL) were separated by sequential ultracentrifugation in a Beckman L-70 preparative ultracentrifuge. ${ }^{23}$ The VLDL was flotated at a density of $1.006 \mathrm{~g} / \mathrm{ml}$ and separated by tube slicing. The density of the subnatant was thereafter raised to $1.063 \mathrm{~g} / \mathrm{ml}$ and the LDL spun to surface; the bottom fraction was then taken to represent HDL. Cholesterol concentration was determined from whole serum and from the lipoproteins by either chemical ${ }^{24}$ or enzymatic (BoehringerMannheim kit, No 187313) method. The results of the chemical method were appropriately corrected to correspond with those of enzymatic assay. Triglyceride concentrations were measured by autoanalyser. ${ }^{25}$

\section{STATISTICAL ANALYSIS}

The significance of differences between the two groups was tested by either unpaired $t$ test or $\chi^{2}$ test with the Yates correction.

\section{Results}

The distribution of risk factors for coronary heart disease was fairly similar at entry for patients and controls (table I). If anything, the patients had higher initial scores for risk factors than the controls as more of them had a history of myocardial infarction or hypertension. The initial mean plasma triglyceride concentration was also higher in the patients than controls, but the difference was not significant. In contrast, more controls than patients were cigarette smokers. The groups did not show any significant differences in the severity of the initial angiographic state assessed by the number and degree of arterial lesions.

The treatment to reduce lipid concentrations resulted in a significant decline in plasma cholesterol and triglyceride concentrations in the patients during the first year, the effect being sustained for the whole seven years of the trial. The average reductions in cholesterol and triglyceride concentrations were $18 \%$ and $38 \%$, respectively. The LDL cholesterol concentration was reduced by an average of $19 \%$, 
and the mean HDL cholesterol concentration was $10 \%$ higher during treatment than at entry. Blood pressure in all the patients was within the normal range at the beginning of and throughout the study. Despite the instructions for reducing weight the mean body weight of the 24 patients who were still alive at seven years increased slowly during the trial, being at the end $4 \%$ higher than the initial value $(p<0.01)$; it increased in 18, was constant in three, and fell in three. Significant changes did not occur in the serum lipid concentrations of the 13 controls still alive at five years. Follow up data were not available for the seven controls who died during the five years of observation.

TABLE I-Baseline characteristics of patients and controls with coronary disease

\begin{tabular}{lll}
\hline \multicolumn{1}{c}{ Risk factor } & $\begin{array}{c}\text { Patients } \\
(\mathrm{n}=\mathbf{2 8})\end{array}$ & \multicolumn{1}{c}{$\begin{array}{c}\text { Controls } \\
(\mathrm{n}=20)\end{array}$} \\
\hline Mean (range) age (years) & $44 \cdot 5(31-56)$ & $45 \cdot 1(30-56)$ \\
No with family history of coronary heart disease & 18 & 14 \\
No with previous myocardial infarction & 14 & 4 \\
No with history of hypertension & 12 & 5 \\
No overweight ( $>120 \%$ ideal body weight) & 5 & 4 \\
No of smokers & 9 & 10 \\
No of ex-smokers & 9 & 6 \\
Mean (SD) cholesterol (mmol/1) & $7 \cdot 70(1 \cdot 34)$ & $7 \cdot 78(1 \cdot 33)$ \\
Mean (SD) triglyceride (mmol/1) & $2 \cdot 79(1 \cdot 43)$ & $2 \cdot 14(1 \cdot 15)$ \\
No with hyperlipidaemia type: & 7 & 9 \\
IIa & 13 & 8 \\
IIb & 8 & 3 \\
IV, V & $1 \cdot 9$ & $2 \cdot 1$ \\
Average No of arterial obstructions of $\geqslant 75 \%$ & & \\
per patient & & \\
\hline
\end{tabular}

TABLE II-Progression of coronary atherosclerosis in patients and controls

\begin{tabular}{|c|c|c|}
\hline & $\begin{array}{l}\text { Patients } \\
(n=28)\end{array}$ & $\begin{array}{l}\text { Controls } \\
(\mathbf{n}=13)\end{array}$ \\
\hline $\begin{array}{l}\text { No (\%) of patients without angiographic } \\
\text { progression } \\
\text { Mean proportional increase in obstruction of } \\
\text { coronary vessels (\%) } \\
\text { No of arterial segments at risk } \\
\text { No }(\%) \text { of arterial segments showing } \\
\text { progression }\end{array}$ & $\begin{array}{c}9(32)^{* *} \\
278 \begin{array}{l}74 \cdot 3^{*} \\
46(16 \cdot 5)^{* *}\end{array}\end{array}$ & $\begin{array}{c}1(8) \\
132^{141 \cdot 1 \dagger} \\
50(38 \cdot 2) \dagger\end{array}$ \\
\hline
\end{tabular}

Significance of difference: $* p<0.05, * * p<0.001$

tAdjusted for seven year follow up.

Angiographic findings-In nine of the 28 patients the coronary lesions did not progress during the seven years of observation. In contrast, the coronary lesions had progressed in all but one of the 13 surviving controls after five years. The difference was significant $\left(p<0.001, \chi^{2}\right.$ test (table II)). Regression of atheromatous lesions was not observed in either group. The patients also showed significantly less progression of coronary lesions when assessed on the basis of the average proportional increase in obstruction (table II). A similar result was also obtained by segment analysis: in the patients 46 of 278 coronary segments at risk showed progression compared with 50 of 132 coronary segments in the controls $\left(p<0.001, \chi^{2}\right.$ test (table II)).

Relation between serum lipid or lipoprotein concentrations during treatment and angiographic findings-Table III presents the mean increase in obstruction and the relative number of arterial segments showing progression separately for patients with an average lipid or lipoprotein concentration during treatment above or below the median value of each variable. The trend for greater progression in the patients with high cholesterol and triglyceride concentrations and high ratios of LDL to HDL cholesterol concentrations compared with the patients with low concentrations was clearly evident, although the difference for triglyceride concentrations did not reach significance. The patients with low HDL cholesterol concentration showed more progression of coronary lesions than those with high HDL cholesterol concentration, whereas LDL cholesterol concentration did not show any relation to angiographic progression. On the other hand, the patients with VLDL plus LDL cholesterol concentrations above the median showed more progression than those with VLDL plus LDL cholesterol concentrations below the median $(21.3 v 12.0 \% \quad(p=0.05$, $\chi^{2}$ test) for segments showing progression). The correlation between proportional increase in luminal narrowing and various lipid variables was significant for HDL cholesterol concentration $(r=-0 \cdot 36 ; p=0 \cdot 05)$. Table IV shows the mean serum lipid and lipoprotein concentrations in patients together with different degrees of increased obstruction seen on angiography. The values were essentially similar for patients showing no progression and for those showing slight progression, whereas the patients with definite progression showed higher cholesterol and triglyceride concentrations but lower HDL cholesterol concentrations than those with less progression, although none of the differences was significant.

TABLE IV-Mean (SD) serum lipid and lipoprotein concentrations in patients divided according to progression of coronary lesions

\begin{tabular}{lccc}
\hline & \multicolumn{3}{c}{ Angiographic progression } \\
\cline { 2 - 4 } & $\begin{array}{c}\text { Absent } \\
(\mathrm{n}=9)\end{array}$ & $\begin{array}{c}\text { Slight } \\
(\mathrm{n}=9)\end{array}$ & $\begin{array}{c}\text { Severe } \\
(\mathrm{n}=10)\end{array}$ \\
\hline Cholesterol (mmol/1) & $6.00(0.54)$ & $6.37(0.69)$ & $6.68(1.42)$ \\
Triglyceride (mmol/1) & $1.58(0.54)$ & $1.62(0.56)$ & $2.01(1.01)$ \\
HDL cholesterol (mmol/1) & $1.16(0.34)$ & $1.15(0.32)$ & $0.97(0.21)$ \\
Ratio of LDL to HDL cholesterol & $3.91(1.25)$ & $3.93(1.61)$ & $5.02(1.80)$ \\
\hline
\end{tabular}

Mortality-Four patients died during the follow up of seven years. One of these deaths was due to a non-cardiac cause. Seven of the controls died during follow up of five years; all deaths were due to cardiac causes. Thus the annual mortality associated with coronary heart disease was $1.5 \%$ in the treated group compared with $7.0 \%$ in the control group $(\mathrm{p}<0.01)$.

Course of anginal symptoms-The favourable effect of treatment on the progression of coronary atheromatosis was reflected in the course of anginal symptoms. During follow up chest pain decreased in nine and remained stable in seven patients compared with two and three controls respectively ( $p<0.05, \chi^{2}$ test). Six of the 24 surviving patients and eight of the 13 surviving controls underwent coronary bypass surgery after the end of the trial. None of the subjects in either group developed a non-fatal myocardial infarction during follow up.

\section{Discussion}

This study was not a randomised controlled trial. The patients were managed with a diet, and most also received drugs, to lower lipid concentrations. The response to treatment varied, but some reduction in serum lipid concentrations was

TABLE III-Progression of angiographic coronary lesions in relation to mean lipid and lipoprotein concentrations during treatment. (Division by median value)

\begin{tabular}{|c|c|c|c|c|c|}
\hline Lipid & $\begin{array}{c}\text { Relation of } \\
\text { median } \\
\text { concentration }\end{array}$ & $\begin{array}{c}\text { Mean proportional increase in } \\
\text { obstruction }(\%)\end{array}$ & $\mathbf{p}^{*}$ & No of segments showing progression ( $\%$ of those at risk) & $\mathbf{p}^{*}$ \\
\hline Cholesterol (mmol/1) & $\left\{\begin{array}{l}>6 \cdot 30 \\
<6.30\end{array}\right.$ & $\begin{array}{r}108 \\
40\end{array}$ & $<0.05$ & $\begin{array}{l}\left.\begin{array}{l}31(22 \cdot 0) \\
15(10 \cdot 9)\end{array}\right\}\end{array}$ & $<0.05$ \\
\hline Triglyceride (mmol/1) & $\left\{\begin{array}{l}>1.60 \\
<1.60\end{array}\right.$ & $\begin{array}{l}92 \\
56\end{array}$ & NS & $\left.\begin{array}{l}27(20 \cdot 1) \\
19(13 \cdot 0)\end{array}\right\}$ & NS \\
\hline HDL cholesterol (mmol/l) & $\left\{\begin{array}{l}>1 \cdot 10 \\
<1 \cdot 10\end{array}\right.$ & $\begin{array}{r}43 \\
105\end{array}$ & NS & $\left.\begin{array}{l}16(11 \cdot 3) \\
30(21 \cdot 9)\end{array}\right\}$ & $<0.05$ \\
\hline Ratio of LDL to HDL cholesterol & $\left\{\begin{array}{l}>4 \cdot 0 \\
<4 \cdot 0\end{array}\right.$ & $\begin{array}{r}107 \\
41\end{array}$ & $<0.05$ & $\left.\begin{array}{l}30(20 \cdot 3) \\
16(12 \cdot 3)\end{array}\right\}$ & NS \\
\hline
\end{tabular}

- Significance by $x^{2}$ test.

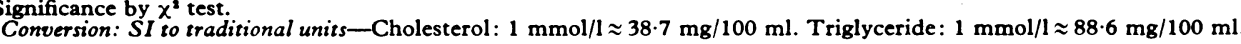


achieved in every case. The controls were men with hyperlipidaemia participating in another prospective study carried out during the same period but aimed at comparing medical and surgical treatments. ${ }^{20}$ The patients in this other study received conventional symptomatic treatment, but they were not given any dietary advice or drugs to reduce lipid concentrations. The patients and controls were largely comparable at entry, having roughly similar levels of risk factors and degrees of coronary atherosclerosis. The comparison of the angiographic progression between the two groups may therefore be thought to be valid, though not ideal.

All clinical and angiographic findings of the present study suggest that treatment of hyperlipidaemia retarded the progression of coronary atherosclerosis. The patients, who received this treatment, differed favourably from the controls in survival, course of anginal symptoms, physical performance, increase in the degree of coronary narrowing, and number of coronary arterial segments showing progression. Any exact comparison of the results with previously published data on the "natural" course of coronary atherosclerosis is difficult because most angiographic follow up studies have been retrospective, of shorter duration, and biased by a high incidence of case selection. The cardiac survival rate of $89 \%$ in seven years in our patients was much higher than that generally reported for patients with coronary disease of two or three vessels. Thus the corresponding figure from a recent study of a large, medically treated group of patients with severe coronary atherosclerosis was about $45 \% \cdot{ }^{26}$ In two prospective angiographic studies the five year survival rates were $69 \%{ }^{27}$ and $80 \% .^{20}$ The proportion of separate arterial segments showing increased obstruction depends on the initial degree of narrowing. ${ }^{27}$ The findings reported so far have in general varied between 20 and 30\% during a five year follow up 22228 ; the figure of $10-13 \%$ in seven years found among our patients who responded well to treatment for hyperlipidaemia compare favourably with these.

The study also showed that the average angiographic progression was least in the patients with the lowest cholesterol, triglyceride, and VLDL plus LDL cholesterol concentrations during treatment and in those with the highest HDL cholesterol concentration. The absence of an association between progression and LDL cholesterol concentration was an unexpected finding that remains without adequate explanation. In follow up studies of patients with coronary disease in whom there is no intervention the association of angiographic progression with hyperlipidaemia has varied. ${ }^{28}{ }^{29}$

The results agree with previous observations on retardation of progression of femoral atherosclerosis by treatment of hyperlipidaemia. ${ }^{12} 1530$ Peripheral atherosclerosis seems to be more amenable to prevention and even to regression of the lesions than coronary disease as significant effects on peripheral atherosclerosis have already been detected within one to one and a half years of the start of treatment to lower lipid concentrations. ${ }^{12} 1530$ Less advanced coronary artery disease may, however, be more responsive to measures to lower lipid concentrations than the coronary disease of the patients in this study, who were all selected on the basis of severe symptomatic coronary atherosclerosis, in which many of the lesions had probably already reached an irreversible stage. The results are, however, encouraging and suggest that treatment to lower lipid concentrations may be more effective in preventing coronary heart disease than the previously disappointing results of some prevention trials have suggested. ${ }^{31}$ Indeed, the present results show that effective treatment of hyperlipidaemia is worth while, even in the stage of secondary prevention of coronary artery disease, and are consistent with the positive result recently reported from an extensive primary prevention trial using cholestyramine. $^{8}$

EAN was supported by grants from the Finnish Medical Research Council (Academy of Finland) and the Yriö Jahnsson Foundation. We thank the patients for their cooperation and Mrs Leena Lehikoinen, Sirkka Runeberg, and Ulla Matilainen for technical help.

\section{References}

Gresham GA. Is atheroma a reversible lesion? Atherosclerosis 1976;23:379-91. Wissler RW. Current status of regression studies. Atherosclerosis Review 1978;3

$213-9$.
Stary HC. Regression of atherosclerosis in primates. Virchows Arch A [Pathol Anat] $1979 ; 383: 117-34$

Miettinen M, Turpeinen O, Karvonen MJ, Elosuo R, Paavilainen E. Effect of cholesterol-lowering diet on mortality from coronary heart disease and other causes. Lancet 1972 ;ii:835-8.

Rosenhamer G, Carlson LA. Effect of combined clofibrate-nicotinic acid treatment in ischaemic heart disease. An interim report. Atherosclerosis $1980 ; 37: 129-38$. Hjermann I, Velve Byre K, Holme I, Leren P. Effect of diet and smoking inter vention on the incidence of coronary heart disease. Lancet 1981 ;ii:1303-10.

May GS, Eberlein KA, Furberg CD, Passamani ER, DeMets DL. Secondary prevention after myocardial in

${ }^{8}$ Lipid Research Clinics Program. The Lipid Research Clinics coronary primary prevention trial results. I. Reduction in incidence of coronary heart disease. cholesterol lowering. $\mathcal{F} A M A$ I $1984 ; 251: 351-74$.

Report from the Committee of Principal Investigators. A co-operative trial in the primary prevention of ischaemic heart disease using clofibrate. Br Heart 1978;40:1069-118

${ }^{0}$ MRFIT Research Group. Multiple risk factor intervention trial: risk factor changes and mortality results. F $A M A$ 1982;248:1465-77.

Ost C-R, Stenson S. Regression of peripheral atherosclerosis during therapy with high doses of nicotinic acid. Scand f Clin Lab Invest [S Suppl] 1968;93:241-5.

12 Blankenhorn DH, Brooks SH, Selzer RH, Barndt R, Jr. The rate of atherosclerosis change during treatment of hyperlipoproteinemia. Circulation 1978;57:355-61. ikkilä EA. Is human atherosclerosis reversible ? In: Miller NE, Lewis B, eds.
Lipoproteins, atherosclerosis and coronary heart disease. Amsterdam: Elsevier/
North-Holland Biomedical Press, 1980:155-64.

Malinow MR. Regression of atherosclerosis in humans: fact or myth ? Circulation $1981 ; 64: 1-3$.

15 Duffield RGM, Lewis B, Miller NE, Jamieson CW, Brunt JNH, Colchester ACF Treatment of hyperlipidaemia retards progression of symptomatic femoral atherosclerosis : a randomized controlled trial. Lancet 1983; ii:639-42.

16 Buchwald H, Moore RB, Rucker RD, Jr, et al. Clinical angiographic regression of atherosclerosis after partial ileal bypass. Atherosclerosis $1983 ; 46: 117-28$.

17 Thompson GR, Myant NB, Kilpatrick D, Oakley CM, Raphael MJ, Steiner RE. Assessment of long-term plasma exchange for familial hypercholesterolaemia. Br Heart $\mathcal{F} 1980 ; 43: 680-8$.

18 Kuo PT, Hayase K, Kostis JB, Moreyra AE. Use of combined diet and colestipol in long-term (7-7 $\frac{1}{2}$ years) treatment of patients with type II hyperlipoproteinemia. Circulation 1979;59:199-211.

18a Brensike JF, Levy RI, Kelsey SF, et al. Effects of therapy with cholestyramine on progression of coronary arteriosclerosis: results of the NHLBI type II coronary Niklilä $E A$ Viikink. $P$, Valle 198 ;69:313-37.

gresion , 58:II-50.

${ }^{20}$ Frick MH, Valle M, Harjola P-T. Progression of coronary artery disease in randomized medical and surgical patients over a 5-year angiographic follow-up. Am 7 Cardiol 1983;52:681-5.

${ }^{21}$ Frick $\mathrm{MH}$, Valle M, Harjola P-T, Korhola O. Changes in native coronary arteries after coronary bypass surgery. Role of graft patency, serum lipids and hypertension. Am $\mathcal{F}$ Cardiol 1975;36:744-50.

22 Bruschke AVG, Wijers TS, Kolsters W, Landmann J. The anatomic evolution of coronary artery disease demonstrated by coronary arteriography. in 256 nonoperated patients. Circulation $1981 ; 63: 527-36$.

${ }^{23}$ Havel RJ, Eder HS, Bragdon JH. The distribution and chemical composition of ultracentrifugally separated lipoproteins in human serum. F Clin Invest 1955 ; 34:1345-53.

24 Huang TC, Chen CP, Wefler V, Raftery A. A stable reagent for the LiebermanBurchman reaction. Application to rapid serum-cholesterol determination. Anal Chem 1961;33:1405-8.

28 Anal Chem $1961 ; 33: 1405-8$. Skeggs LT, ed. Automation in analytical chemistry. New York: Mediad, 1966 : 341-4 (Tech. Automation in

${ }^{26}$ Proudfit WJ, Bruschke AVG, MacMillan JP, Williams GW, Sones FM Jr Fifteen year survival study of patients with obstructive coronary artery disease. Circulation 1983;68:986-97.

" Palac RT, Hwang MH, Meadows WR, et al. Progression of coronary artery disease in medically and surgically treated patients 5 years after randomization. Circulation $1981 ; 64:$ II-17-21.

3 Brown BG, Bolson EL, Dodge HT. Arteriographic assessment of coronary atherosclerosis. Review of current methods, their limitations, and clinical applications. Arteriosclerosis $1982 ; 2: 2-15$.

10 Roskamm H, Gohlke H, Sturzenhofecker $P$, et al. Myocardial infarction in the young: coronary morphology, risk factors, long-term prognosis, and progression

30 Olsson AC, Carlson LA, Erikson U, Helmius G, Hemmingson A, Ruhn G Regression of computer estimated femoral atherosclerosis after pronounced Regression of computer estimated femoral atherosclerosis after pronounced serum lipid

"Oliver MF. Serum cholesterol-the knave of hearts and the joker. Lancet 1981 ;ii: 1090-5.

(Accepted 24 April 1984) 